\title{
Acute Mesenteric Ischemia after Recurrent Embolic Stroke
}

Hiroaki Oguro* and Shuhei Yamaguchi

Department of Neurology, Shimane University School of Medicine, 89-1 Enya-cho, Izumo 693-8501, Japan

\section{Abstract}

We report the case of a 76-year-old woman with acute mesenteric artery ischemia and atrial fibrillation after recurrent embolic strokes. She had sudden severe abdominal pain and vomiting, and was admitted to the hospital in coma and shock state. Accompanied with metabolic acidosis and multiple organic failures, she died at 13 hours after the onset. Pathological examination revealed that acute thrombotic occlusion of superior mesenteric artery, splenic artery and right celiac artery. The occluded arteries pathologically showed progressive arteriosclerotic change and secondary multiple thrombi. This case demonstrated that atrial fibrillation with recurrent embolic stroke could cause mesenteric artery ischemia, progressive thrombi of abdominal viscera and sudden death.

\section{Introduction}

Acute mesenteric artery ischemia is relatively rare acute abdominal vascular event caused by aging, atrial fibrillation, aortic atheroma and infectious endocarditis $[1,2]$. The incidence is 5.3 per 100,000 inhabitants yearly, and its mortality is $60 \%$ to $80 \%$ [3]. It counts $0.4 \%$ in patients with acute abdominal pain. Acute mesenteric ischemia is a life-threatening emergency that requires early diagnosis, intervention to restore mesenteric blood flow and resection of the necrotic bowel tissues by surgical treatments [4]. This report describes a patient with atrial fibrillation and recurrent stroke which rarely cause acute mesenteric ischemia and sudden death with multiple organic failures and lower limb ischemia. After stroke onset sudden abdominal pain and followed by shock state is suggestive of diagnosis of acute mesenteric ischemia.

\section{Case Report}

A 77-year-old Japanese woman with atrial fibrillation and hypercardiomyopathy was treated by a cardiologist. Two months earlier she had embolic strokes of the right middle cerebral artery, then oral anticoagulation by warfarin had been administered to prevent recurrent stroke. In spite of controlling anticoagulant therapy by warfarin within two months, the prothrombin time-international normalized ratio(PT-INR) with 10 times analysis, three of ten times apparently showed inadequate values below the therapeutic range $(0.93,1.00$ and 1.33). The CT scan of the head showed hemorrhagic infarction caused by recanalization after occlusion of the right middle cerebral artery (Figure 1). The echocardiography showed left atrial spontaneous echo contrast on previous stroke onset. On the $66^{\text {th }}$ day after the stroke onset, she experienced severe nausea, vomiting and abdominal pain in the morning, and delivered to our hospital. On arrival at our emergency department, she was coma. Her blood pressure could not be measured, and heart rater pulse was 124 bpm with irregular rhythm. Respiratory rate was $42 / \mathrm{min}$ and her temperature was 34.2 degrees. Right lower limb showed pale color and hemorrhagic purpura with pulseless iliac artery. Her abdomen showed rebound tenderness and no bowel sound.

Laboratory examination showed, glutamic oxaloacetic transaminase (GOT) of $1389 \mathrm{IU} / \mathrm{l}$, glutamic pyruvic transaminase (GPT) of 929 IU/l, lactate dehydrogenase (LDH) of $5366 \mathrm{IU} / \mathrm{l}$, amylase of 547 $\mathrm{IU} / \mathrm{l}$, and arterial blood gas analysis showed metabolic acidosis with a $\mathrm{pH}$ of 7.185 , a partial pressure of carbon dioxide $(\mathrm{PaCO} 2)$ of $11.3 \mathrm{mmHg}$ and partial pressure of oxygen $(\mathrm{PaO} 2)$ of $194.5 \mathrm{mmHg}$ with oxygen inhalation by a reserve mask. The PT-INR was 1.85 . Serum homocysteine, Protein C, Pretein S and Antithrombin III
(AT III) was normal. The plain CT of the abdomen did not showed any hemorrhage and ascites. We diagnosed multiple embolism and mesenteric ischemia and started heparin and gabexate mesilate. The function of liver and pancreas rapidly worsened. At 13 hours after the onset, she died of shock with metabolic acidosis and multiple organ failure.
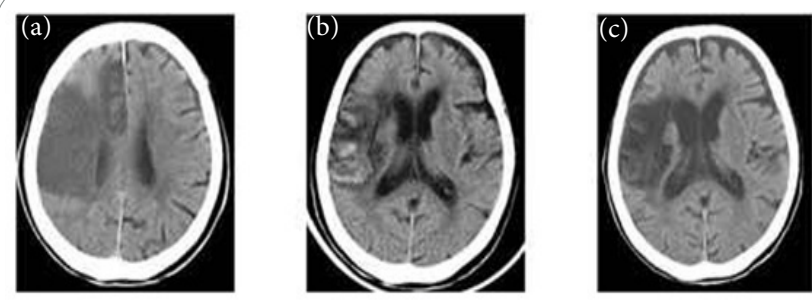

Figure 1: The plane CT image, (a) first stroke onset, (b) 15th day from first onset, (c) 66th day from first onset.

Pathological examination revealed the thrombus was found at celiac trunk (Figure 2a), superior mesenteric artery (SMA) (Figure $2 \mathrm{~b})$, splenic artery, right renal artery and right external iliac artery. Several visceral organs revealed necrotic change for pancreas, atrophic degeneration for liver and hemorrhage for spleen. These three organs also showed acute ischemic necrosis with rapid progression. Figure 3 shows thrombus of splenic artery which was formed by several pathological changes, white thrombus, coagulated and organized one. Neoplastic capillary had grown in organized lesion of thrombus. Macro and microscopic findings of kidney showed mixed pathological changes, partial infarction with wedge shaped area, relative recent hemorrhagic infarction and ischemic necrosis with long-term periods. Occluded vessels revealed arteriosclerotic change and mixed thrombi formed for a long time. Homogenous coagulant necrosis by complete ischemia and necrotic depletion of the tubulus by long-term ischemia were simultaneously seen in the same kidney. After the necrotic change, glomerular reperfusion and hemorrhage

"Corresponding Author: Dr. Hiroaki Oguro, Department of Neurology, Shimane University School of Medicine, 89-1 Enya-cho, Izumo 693-8501, Japan; E-mail: oguro@med.shimane-u.ac.jp

Citation: Oguro H, Yamaguchi S (2015) Acute Mesenteric Ischemia after Recurrent Embolic Stroke. Int J Gastroenterol Disord Ther 2: 117. doi: http:// dx.doi.org/10.15344/2393-8498/2015/117

Copyright: (C) 2015 Oguro et al. This is an open-access article distributed under the terms of the Creative Commons Attribution License, which permits unrestricted use, distribution, and reproduction in any medium, provided the original author and source are credited. 
had occurred. The kidney showed variable histological changes in terms of vascular territory and time course.



Figure 2: (a) Thrombotic occlusion of celiac trunk and superior mesenteric artery, (b) Thrombosis in superior mesenteric artery. (a)

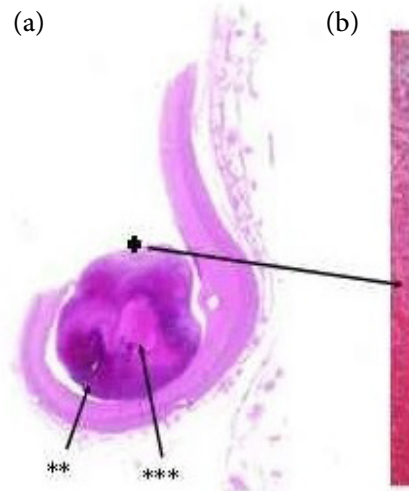

(b)

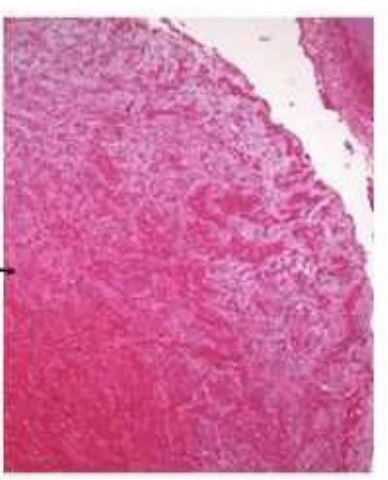

Figure 3: (a) Thrombus in splenic artery contains organized thrombus ${ }^{\star}$, coagulation ${ }^{\star *}$ and white thrombus ${ }^{* * *}$, (b) Neoplastic capillary in organized thrombus with enlarged image.

\section{Discussion}

Systemic embolism followed by the formation of a thrombus in the left atrium causes multiple infarcts in viscera and limbs. Although they are less common and more often asymptomatic, they should be treated by warfarin to prevent secondary systemic embolism [5].We could not find intracardiac thrombus in autopsy. However, left atrial spontaneous echo contrast suggested multiple thromboembolism occurred toward many organs from the left atrium. The large cerebral infarction and renal infarction might suggest former tendency of thrombus formation.

There are several mechanisms in peripheral atherosclerosis plaque formation and thromboembolism. Severe atherosclerosis plaque formation causes vascular obstruction. Emboli originating from the fibrillating atrium and ruptured plaque on large artery causes acute occlusion of distal arteries. Activated coagulation systems also cause mesenteric ischemia. In the Denmark study patients with peripheral arterial thromboembolism caused by atrial fibrillation occurred $29 \%$ in the mesenteric arteries, $2 \%$ in the renal artery and $61 \%$ in the extremities, and sometimes more than one site at the same time [6]. Generalized atherosclerosis is also major risk for acute mesenteric ischemia; Mishima Y reported 27\% of 162 cases has general atheroma [7]. In our case atherosclerosis of aorta was moderate with no luminal thrombus, and the bifurcation of major arteries from the trunk occluded with thrombus. So we could speculate multiple shower emboli occurred from the left atrial thrombi, which had gradually grown up for long periods and finally caused embolic occlusion stenosis of arterial vessels. In the descending aorta, partial thrombus and atheromatous changes were seen. Irregular formation of thrombus and irregular thickness of aortic intima inform us of organic change of chronic thromboembolism.

Left atrial spontaneous echo contrast in the preceding echocardiography before 2 months, and multiple infarctions in cerebral and bilateral kidney suggest sudden multiple thromboembolism. This patient had a relative high risk of recurrent stroke because her CHADS2 index was 4 with age 75 years or older, prior stroke history, heart failure [8]. The stroke rate per 100 patient-years is 8.5 (95\% CI, 6.3-11.1) for a score of 4 [9]. Although this patient was taking warfarin, the PT-INR at admission was less than 2.0 below the therapeutic range. Hylek reported that among patients who were taking warfarin, the PT-INR of less than 2.0 as compared with 2.0 or greater increased the odds of a severe stroke in odds ratio 1.9 [10]. She had been in a high risk state and needed strict anticoagulant control by warfarin.

Three visceral organs, pancreas, liver and spleen showed rapid progression in ischemic necrosis. Various renal infarctions suggest repetition of thromboembolism in renal artery. The various findings of thrombus in splenic artery reveal that it took long term for the thrombus formation. We can also speculate the possibility of embolism in branch arteries from aorta. Slow progressive of atherosclerosis allows development of collateral circulation, but last remaining artery occlusion causes bowel ischemia [4]. This case might also have some collateral flows because of progressive thrombus formation in splenic artery. Hachiska et al. reported that an autopsy case with SMA ischemia secondary to embolic stroke in Japan. This case reveals old infarction focus in cerebrum and kidney similar to our case [1]. Acosta $\mathrm{S}$ et al. reported autopsy findings in 213 occlusion of SMA. 83 cases $(39 \%)$ revealed synchronous emboli in other arteries, renal artery emboli were 50(23\%), common hepatic artery was $12(5 \%)$, iliac artery was $27(12 \%)$ and splenic artery was $28(13 \%)$. And they also reported that $93(43 \%)$ cases have old brain infarction [2]. Infarctions in multiple major organs simultaneously occurred with acute mesenteric ischemia. Similarly our case had simultaneous emboli in all of above mentioned arteries, so this was a severe thromboembolic occlusion case. This case was rare in pathological findings of simultaneously occluded many visceral arteries and progressive formation of thromboembolus in splenic and renal arteries.

Warfarin and other Vitamin $\mathrm{K}$ antagonists have a narrow therapeutic range, long half-lives and many drug-drug interactions. Dosing of these drugs can be unpredictable due to genetic, diseaserelated, and environmental factors. The new oral factor Xa inhibitor, edoxaban for the prevention of stroke and systemis emboli in patients with atrial fibrillation have favorable pharmacokinetic profiles with minimal-to no requirements for therapeutic monitoring [11]. It will be effective for this case with difficulty in controlling anticoagulant.

\section{Conclusion}

Old age, atrial fibrillation and post stroke state below the therapeutic range of anticoagulation might cause thromboembolic superior mesenteric artery occlusion with a fatal outcome. Whenever examining the old patient with severe unexplained abdominal pain, it is necessary to keep the diagnosis of mesenteric artery ischemia in mind.

\section{Competing Interests}

The authors have no competing interests with the work presented in this manuscript. 
Citation: Oguro H, Yamaguchi S (2015) Acute Mesenteric Ischemia after Recurrent Embolic Stroke. Int J Gastroenterol Disord Ther 2: 117. doi: http://dx.doi. org/10.15344/2393-8498/2015/117

\section{Author Contributions}

Both the author substantially contributed to the study conception and design as well as the acquisition and interpretation of the data and drafting the manuscript.

\section{References}

1. Hachisuka A, Ogata T, Yasaka M, Momosaki S, Okada Y (2009) An autopsy case revealing acute mesenteric ischemia during treatment for embolic stroke. Jpn J Stroke 31: 233-237.

2. Acosta S, Ogren M, Sternby NH, Bergqvist D, Björck M (2005) Clinical implications for the management of acute thromboembolic occlusion of the superior mesenteric artery: autopsy findings in 213 patients. Ann Surg 241: 516-522.

3. Acosta S, Björck M (2003) Acute thrombo-embolic occlusion of the superior mesenteric artery: a prospective study in a well defined population. Eur $\mathrm{J}$ Vasc Endovasc Surg 26: 179-183.

4. Oldenburg WA, Lau LL, Rodenberg TJ, Edmonds HJ, Burger CD (2004) Acute mesenteric ischemia: a clinical review. Arch Intern Med 164: 10541062.

5. Andersen LV, Vestergaard P, Deichgraeber P, Lindholt JS, Mortensen LS et al. (2008) Warfarin for the prevention of systemic embolism in patients with non-valvular atrial fibrillation: a meta-analysis. Heart 94: 1607-1613.

6. Frost L, Engholm G, Johnsen S, Møller H, Henneberg EW, et al. (2001) Incident thromboembolism in the aorta and the renal, mesenteric, pelvic, and extremity arteries after discharge from the hospital with a diagnosis of atrial fibrillation. Arch Intern Med 161: 272-276.

7. Mishima Y (1988) Acute mesenteric ischemia. Jpn J Surg 18: 615-619.

8. van Walraven C1, Hart RG, Wells GA, Petersen P, Koudstaal PJ, et al (2003) A clinical prediction rule to identify patients with atrial fibrillation and a low risk for stroke while taking aspirin. Arch Intern Med 163: 936-943.

9. Gage BF, Waterman AD, Shannon W, Boechler M, Rich MW, et al. (2001) Validation of clinical classification schemes for predicting stroke: results from the National Registry of Atrial Fibrillation. JAMA 285: 2864-2870.

10. Hylek EM, Go AS, Chang Y, Jensvold NG, Henault LE, et al. (2003) Effect of intensity of oral anticoagulation on stroke severity and mortality in atrial fibrillation. N Engl J Med 349: 1019-1026.

11. Piccini JP, Lopes RD, Mahaffey KW (2010) Oral factor Xa inhibitors for the prevention of stroke in atrial fibrillation. Curr Opin Cardiol 25: 312-320. 